\title{
A GEANT4 Web-based Application to Support Intra- Operative Electron Radio-Therapy using the European Grid Infrastructure
}

\author{
Carlo Casarino, Giorgio Russo, Giuliana Carmela \\ Candiano \\ IBFM CNR - LATO \\ Cefalù - Italy
}

Giovanni Borasi

University of Milano Bicocca, Milan - Italy

$$
\begin{aligned}
& \text { Maria Carla Gilardi } \\
& \text { IBFM CNR - LATO } \\
& \text { Cefalù - Italy }
\end{aligned}
$$

University of Milano Bicocca, Milan - Italy

San Raffaele Scientific Institute, Milan - Italy

\author{
Giuseppe La Rocca, Roberto Barbera \\ Italian National Institute of Nuclear Physics, Division of \\ Catania - Italy
}

Department of Physics and Astronomy of the University of Catania - Italy

Cristina Messa

IBFM CNR - LATO

Cefalù - Italy

University of Milano Bicocca, Milan - Italy

San Gerardo Hospital, Monza - Italy

\author{
Gianluca Passaro \\ Consortium COMETA, Catania, Italy
}

\begin{abstract}
Radiotherapy techniques permit to deliver ionizing radiations (X-rays, photons, electrons, protons, etc) inside cancerous tissues to kill the abnormal-cells. Radiotherapy related activities, as the optimization of the therapeutic radiation dose to the patient, worker radioprotection, performance controls and technical innovations of linear accelerators, are strongly based on the ability to predict the dose distribution. Monte Carlo simulations are the most accurate tools in this field but, unfortunately, they require large computing power to achieve accurate results in reasonable times. In the last years, advanced cancer treatment clinical and research communities have adopted e-Infrastructures to reduce this gap. The present paper reports on the developments of a computing facility for helping physicians, radiotherapists and medical physical in using modern R\&E networking and distributed resources to address some technical and clinical Intra-Operative Electron Radio-Therapy (IOERT) needs (e.g. the design of the linear accelerator collimation system or the optimization of the patient therapeutic dose distribution).
\end{abstract}

Keywords- IOERT, Monte Carlo simulation, GEANT4, Distributed Computing Resources, Catania Science Gateway framework.

\section{INTRODUCTION}

Radiotherapy is one of the most successful non-invasive methodology for the treatment of different types of cancers.
This technique consists in delivering ionizing radiations (Xrays, photons, electrons, protons, etc) inside cancerous tissues to control or kill abnormal-cell growth. Radiation therapy normally has also several applications in non-malignant conditions, such as the treatment of trigeminal neuralgia, acoustic neuromas, severe thyroid eye disease, the prevention of keloid scar growth, vascular restenosis, and heterotopic ossification. The use of radiation therapy in non-malignant conditions is limited partly by worries about the risk of radiation-induced cancers. In addition, radiation therapy can also be used as part of curative therapy, to prevent tumor recurrence after surgery to remove a primary malignant tumor (for example, early stages of breast cancer).

From the medical perspective, the radiation of the cancer cell aim to damage its DNA content and leads rapidly to its death since no mechanisms to auto-recover from a damage are available in the cancer cells. Another important aspect which has to take into account when we talk about radiotherapy is the radio-protection of tissues not affected by the cancer. To spare normal tissues, such as skin or organs, some different technics are used such as: shape the beams during the irradiation, consider many entrance points, fluence modulation, or, in the intraoperative treatment, shift the normal tissue and irradiate the tumor directly. Thanks to this different technics it is possible to guarantee a much larger absorbed dose level in the targeted tissues and protect the normal tissues. 
The rapid development and adoption of radiotherapy techniques have been possible thanks to the introduction a new generation of linear accelerators (often shorted to linac) that greatly increases the velocity of charged subatomic particles or ions by subjecting the charged particles to a series of oscillating electric potentials along a linear beam-line. The design of a linac depends on the type of particle that is being accelerated: electrons, protons or ions. Linac range in size from a cathode ray tube (which is a type of linac) to the 2-mile (3.2 $\mathrm{km}$ ) long linac at the SLAC National Accelerator Laboratory in Menlo Park, California.

Computer simulation of radiation transport and subsequent dose distribution estimate is of fundamental importance, both in clinical and research activities. In the clinical perspective some commercial software, such as Therapeutic Planning Systems (TPS), are commonly in use to plan, approve and in situ verify, patient radiotherapy sessions. TPS scope is to optimize the dose distribution release in the treatment zone and implement the healthy tissues protection. Unfortunately these tools, often, offer unsatisfactory accuracy dose calculation methods, especially in inhomogeneous regions as soft tissue bone boundaries [1]. Moreover there are others clinical activities, involving medical physicists, where simulations may be a support in the development of procedures for the verification of the linac specifications [2] or impractical activities where the radiation transport simulations are irreplaceable, as well adjustments in radiation shielding room design [3], or even impossible, when direct measurements of physical quantities are conceptually not executable as in the case of energy of the particles escaped from the head accelerators [4].

In radiotherapy research, linac manufacturer designers and medical physics study the best solution to generate and collimate the radiation beam and/or to optimize the patient radioprotection. In this context, Monte Carlo simulation method offers the most exact software tool to calculate radiation transport and energy deposition but, unfortunately, require large computing power to achieve accurate results in reasonable times [1]. In fact, particles transports (histories) are processed sequentially and to achieve a correct statistical uncertainty in dose values (in most case within $2 \%$ the experimental values) the number of histories may increase up to $10^{8}$. In these conditions, a single standard CPU (3 GHz) can take even some months to complete a single simulation.

On the other hand, useful (per simulation) times may vary from few hours, as in the case of a TPS, to some days for company and research requests.

To improve simulation efficiency, in the last three decades, the direct synergy between scientific-medical community and linac manufacturers-commercial vendors has produced many alternative no MC solutions, as convolution/superposition methods or approximate, as MC simulation associated with variance reduction techniques [1,5]. Another innovative and promising expedient it is represented by Graphical Processing Unit (GPU) implementation of MC methods. Using the Single Instruction Multiple Data structure presents on the graphic board, the same function may be execute many times simultaneously allowing to process in parallel several histories [6].

However, the best reliable and tested solution is to use the "raw" Monte Carlo method in distributed computing environment. Parallelized computation, in fact, offers to serial processing a natural and more appropriate practical support. A time-heavy simulation (of $\mathrm{N}$ histories) may be splitted in $\mathrm{N}$ ' smaller simulations each one executed on different CPU. For example, a Grid infrastructure offering 200 CPU core for user, permits then to reduce computing-times of a factor of 200 $\left(\mathrm{N} / 200=N^{\prime}\right)$. To address the problematic described above and support a new and advanced radiotherapy technique, the iort_therapy application, has been developed.

The outline of this paper is as follow: in section II we provide IOERT. In section III we will introduce some background information about the iort_therapy application developed by researchers of the IBMF CNR-LATO $[7,8]$. In Section IV an overview of the reference model used for implementing a Science Gateway, which is now used by physicians, radiotherapists and medical physical to address some technical and clinical Intra-Operative Electron RadioTherapy (IOERT) needs, will be described. Finally, we will report about some outcomes and future prospective.

\section{THE INTRA-OPERATIVE ELECTRON RADIOTHERAPY}

The Intra-Operative Electron Radiotherapy (IOERT) is a technique that allows treatment of the cancerous cells directly in the operating theatre after the surgeon have removed the tumor tissues [9]. The high ionizing beam is delivered through special cylindrical applicators positioned during the surgery. The protection of internal normal tissue from radiation leakage is a critical point [4]. In breast treatment, for example, it involves the surgeon positioning a shielding metal disc between the deep face of the patient's residual breast and the pectoral muscle.

For IOERT dedicated and mobile accelerators, such as NOVAC7 (NRT, Aprilia, Italy) [10], Liac (Sordina SpA, Italy) [11], and Mobetron (IntraOp Medical, Inc. Santa Clara, CA) [2], are employed . The main characteristics of these machines relies on the capability to be placed near the patient's bed, the orientation of the beam in different directions, and the delivery of very high dose rates compared to traditional linear accelerators. With the availability of these mobile electron linear accelerators, IOERT procedures have become widespread, at least in major clinical centers, giving the possibility that greater numbers of patients can be treated.

NOVAC7 produces electron beams of 4, 6, 8, and $10 \mathrm{MeV}$ nominal energies to perform treatments at different tissue depths. Applicators (collimators) with different diameters from 3 to $10 \mathrm{~cm}$ are available. The field of radiation is collimated by cylindrical perspex applicators with different diameters and tilt angles. Applicators are available with diameters ranging from 3 to $10 \mathrm{~cm}$ and are either parallel $\left(0^{\circ}\right)$ or beveled $\left(15^{\circ}, 22.5^{\circ}, 30^{\circ}\right.$ and $45^{\circ}$ ). The collimation system is of a hard docking type: the applicators are rigidly attached to the accelerator. Each collimator is made up of two parts: the upper applicator, that is fixed to the radiant head, and the lower applicator, that is placed on the surface to radiate. To perform the treatment, the 
radiant head is slowly moved to place the upper and lower part of collimator in line; a ring allows the two parts to be coupled. Each collimator corresponds to a Source Skin Distance (SSD) of $80 \mathrm{~cm}$ except for the collimator of $10 \mathrm{~cm}$ of diameter that corresponds to a SSD of $100 \mathrm{~cm}$. In figure 1, the NOVAC7 is shown in the surgery ready for operation. The collimation system starts from the end of the accelerator head. The latter acts as the primary collimation system. Connected to this is the secondary collimator, consisting of a larger PMMA cylinder that includes a fixed adaptor and a final collimator.

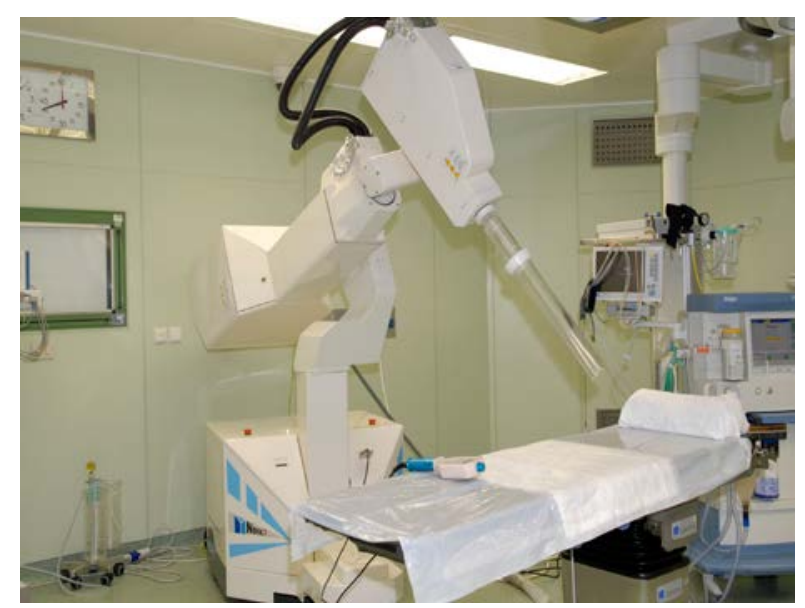

Fig. 1. The mobile linear electron accelerator NOVAC7 in the surgery room.

\section{THE GEANT4 - IORT THERAPY APPLICATION}

To contribute to the study of intra-operative procedures and to the optimization of the machine, we developed a specific Monte Carlo (MC) application, “iort_therapy", using the GEANT4 toolkit [12]. Today it represents one of the advanced examples of the official GEANT4 release (9.5 version) and it can be freely downloaded [13].

The application simulates the electron beam and the collimation system of the NOVAC7 and addresses several technical and clinical issues related to the IOERT technique such as: the design and optimization of the collimation system; the study of patient radio-protection aspects; the optimization of the therapeutic dose distribution [4] and the development of procedures for verification of the linac specifications [14].

The application allows the calculation of dose distribution in water or in other materials. Moreover, it gives the possibility to choose between different clinical setups and to optimize radio-protection of normal tissues. Using macro commands, the user can easily select the appropriate collimator, the phantom and detector characteristics, the configuration of the shielding disc employed in breast treatments, the initial conditions of the electron beam (in particular, position and angular distributions), and the appropriate physics list, i.e., the command list for the physical processes.

In Fig. 2 are shown typical critical situations when the shielding disc is incorrectly positioned (misaligned and tilted) respect the collimation system, to reproduce potential erroneous clinical set-up. In these situations the healthy tissues are exposed to dangerous ionizing radiations.

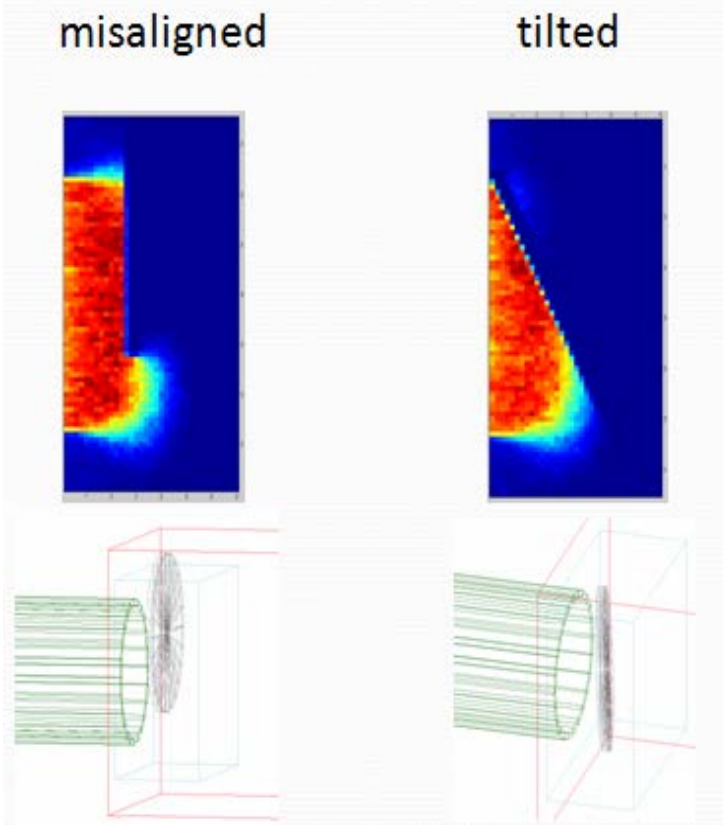

Fig. 2. 2D dose distribution inside the water phantom (reproducing tissues) using a $10 \mathrm{MeV}$ beam collimated with the collimator $10 \mathrm{~cm}$ diameter when the shielding disc is errouneously misaligned and tilted respect the beam collimation system . The ionizing electron beam comes from left to right.

Figure 3 shows the Graphical User Interface (GUI) of iort_therapy. The GUI is implemented with QT4 libraries. By interactive windows (Help, Commands, Command outputs and History) the geometric and physical characteristics of the clinical setup are chosen and visualized. On the right side, the smaller tube represents the accelerator head and includes the exit window, the monitor chamber and the primary collimation system. The larger and longer tube is the second collimation system. The water phantom (simulating patient tissues) is represented by the larger box. It includes a smaller box representing the sensitive detector (70 mm depth $\mathrm{x} 150 \mathrm{~mm} \times$ $150 \mathrm{~mm}$ surface). The shielding disc is positioned in the middle of this structure. To provide a relative error comparable to that obtained experimentally, $10^{8}$ histories per simulation must be generated.

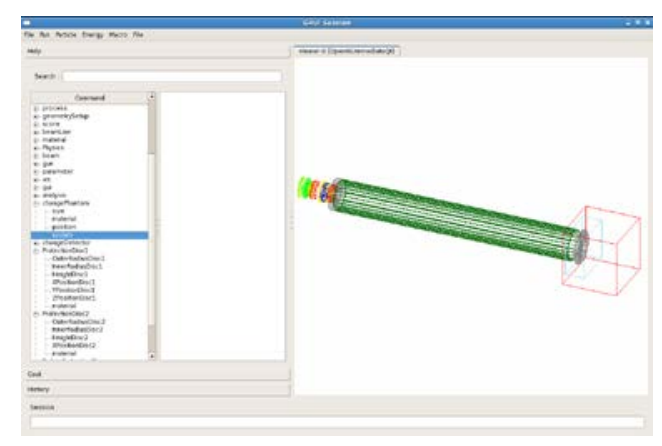

Fig. 3. Graphical User Interface (GUI) of the Geant4 example iort_therapy 
From a technical point of view the application is embarrassingly parallel and needs a prior-installation of Geant4 as well as some additional libraries. The typical output file produced by the tool is a dose distribution in a volume (sensitive detector) of $300 \times 300 \times 140$ voxels. The size of output files vary from few MBs to tens of GBs. The application's workflow is a highly computing demanding problem. On a single CPU (with $3 \mathrm{GHz}$ core) it would require about 200 CPU days to produce the dose distribution with the required precision. The same Monte Carlo computation must also be repeated many times starting from the same input file which contains the macro.

\section{THE REFERENCE MODEL}

This section describes the present status of the IOERT Science Gateway which has been developed by the INFN of Catania for helping physicians, radiotherapists and medical physical to use the pan-European GÉANT network, which operates at speed of up $100 \mathrm{Gbps}$, and the computing facilities of the COMETA Consortium[15] to optimize the patient's radioprotection and/or set up the best collimation system configuration to deliver a high dose to the target tissues.

The IOERT Science Gateway is based on the Catania Science Gateway framework [16] which has been successfully adopted in the context of several EU funded projects such as DECIDE [17], EUMEDGRID-Support [18], GISELA [19], CHAIN [20] and INDICATE [21]. In a nutshell, the Science Gateway uses JSR 286 standards (also known as “portlet 2.0”) to develop advanced tools that can be re-used and combined for resolving different and complex problems and Liferay as a portlets container. The access to the Science Gateway relies on the use of Identity Federations based on the SAML 2.0 standard and on its implementation done by Shibboleth [22] and SimpleSAMLphp [23]. A “catch-all” Identity Federation to gather all the users who do not already belong to any federations and a special IdP that allow people to get authenticated with the same credentials they already have with the most common and populated social networks (Facebook, Google+, LinkedIn, Twitter, Windows Live and Yahoo!) are also supported. The following authorization mechanism makes possible to simplifying the access to the infrastructure and widening its user base. The access to the underlying computing infrastructure relies on the SAGA standard [24] and on its JSAGA implementation. A specific JSAGA Adaptors is used to run Monte Carlo simulations on the COMETA computing facilities. For the authentication point of view, all the grid transactions are secured using a robot certificate installed on a eToken PRO 64KB USB smart card plugged on a dedicated server which is in charge to generate, using PKCS\#11 and JAX-RS standards, proxy certificates for the users. Last but not least, the IORT Science Gateway has been implemented in order to be fully complaint with the strict rules of the EGI VO Portal [25] and EGI Grid Security Traceability and Logging Policy [26] policies. The reference model of the IORT Science Gateway is shown in figure 4.

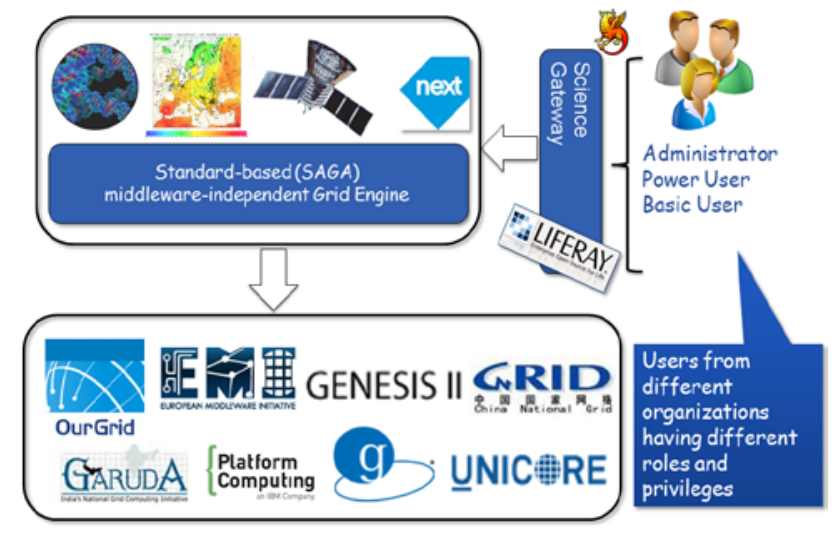

Fig. 4. The reference model for the IOERT Science Gateway

The IOERT Science Gateway is an interdisciplinary work that involves experts belonging to different scientific areas. The main purpose of the Science Gateway is to build a unique entry point for physicians, radiotherapists and medical physical needing to optimize the patient's radioprotection and/or set up the best collimation system configuration to deliver a high dose to the target tissues. When users register to the Science Gateway, they are mapped to valid Grid users so that, through a single sign-on mechanism, they can use the portal services and the underlying computational and storage resources. In fig. 5 is shown a standard JSP page and the relative logic portlet that has been implemented for collecting the input parameters and send a bunch of Monte Carlo simulations on the computing facilities.

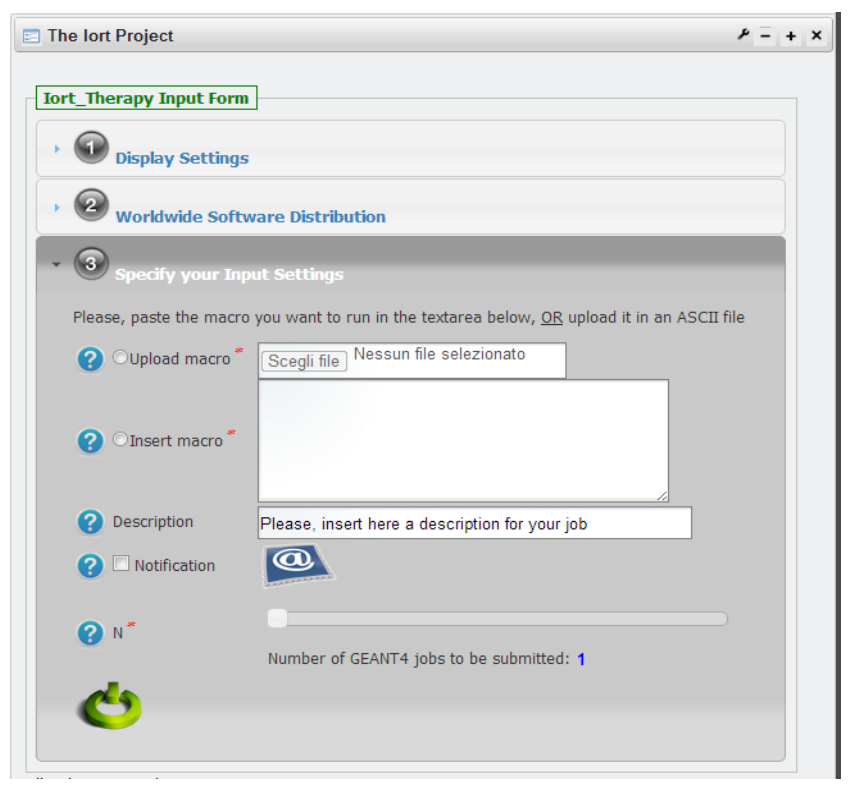

Fig. 5. The IOERT input and job submission page

The input page consists of several input fields with, at the bottom, a link to start the running of the Monte Carlo simulations. Once the simulations have been submitted, users can follow its status and download its output at the end through the links of the MyWorkspace portlet which appears on the left side of the browser when users are signed in. The MyWorkspace portlet aims to handle users' jobs and data 
through the respective and self-explaining functions MyJobs and MyData. As an example, figure 6 shows a typical view of MyJobs.

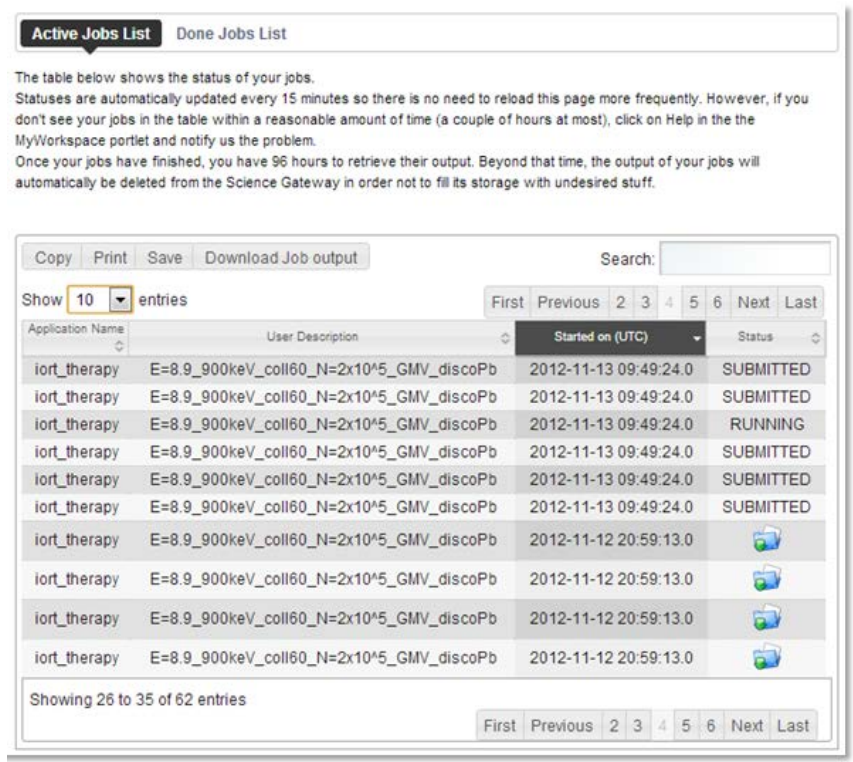

Fig. 6. MyworkSpace portlet showing the list of IOERT simulations

\section{OUTLOOKS}

Thanks to the advanced R\&E networking and the computing facilities of the COMETA consortium, the iort_therapy application produces several $\mathrm{MB}$ of compressed data for each simulation reducing the consumed computing time to $10 \mathrm{CPU}$ hours per run. The raw data generated are then refined offline using some MATLAB sub-routines. In figure 7 is shown the Wall Clock time consumed by the application starting from Oct 2012 when the application started to run in production.

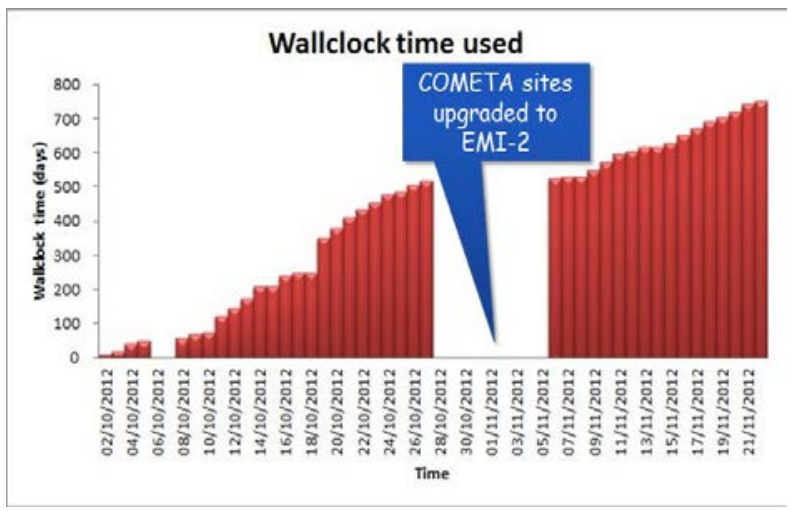

Fig. 7. WallClock time consuded by the iort_therapy application from Oct to Nov 2012

As it is shown by the figure the trend is increasing. With this data rate the application is now able to address some of the Companies and Research Community requirements. As a future prospective, a new iort_therapy version is under development for letting physicians to use this application in hospitals.

\section{REFERENCES}

[1] I. J. Chetty, B. Curran , J. E. Cygler, J. J. DeMarco, G. E. Bruce, A Faddegon, I. Kawrakow, P. J. Keall, H. Liu, C. M. Charlie Ma, D. W. O. Rogers, J. Seuntjens, D. Sheikh-Bagheri, J.V. Siebers. Report of the AAPM Task Group No. 105: Issues associated with clinical implementation of Monte Carlo-based photon and electron externa beam treatment planning. Med. Phys. vol. 34, pp. 4818-4853, December 2007

[2] Mills M D, Fajardo L C, Wilson D L, Daves J L and Spanos W J 2001 Commissioning of a mobile electron accelerator for intraoperative radiotherapy Am. Coll. Med. Phys. 2 121-130

[3] National Council on Radiation Protection and Measurement. Structural Shielding Design and Evaluation for Medical Use of X-Rays and Gamma-Rays up to $10 \mathrm{MeV}$ (NCRP 49). Office of the Federal Register. U.S.A. 1976

[4] Russo G, Casarino C, Arnetta G, Candiano G, Stefano A, Alongi F, Borasi G, Messa C, Gilardi MC. Dose distribution changes with shielding disc misalignments and wrong orientations in breast IOERT: a Monte Carlo - GEANT4 and experimental study. Journal of Applied Clinical Medical Physics, 2012; 13(5):1526-9914

[5] P. Downes, G. Yaikhom, J.P. Giddy, D.W. Walker, E. Spezi and D.G. Lewis. High-performance computing for Monte Carlo radiotherapy calculations. Phil. Trans. R. Soc. vol. 367, pp. 2607-2617, May 2009.

[6] L. Jahnke, J. Fleckenstein, F. Wenz and J. Hesser. GMC: a GPU implementation of a Monte Carlo dose calculation based on Geant4 Phys. Med. Biol. vol.57, pp. 1217-1229, February 2012.

[7] CNR-IBMF. Available from: www.ibfm.cnr.it

[8] LATO HSR Giglio. Available from www.polooncologicocefalu.it/

[9] Veronesi et al 2001 A preliminary report of intraoperative radiotherapy (IORT) in limited-stage breast cancers that are conservatively treated Eur. J. Canc. 37 2178-2183.

[10] Pimpinella M, Mihailescu D, Guerra A S and Laitano R F 2007 Dosimetric characteristics of electron beams produced by a mobile accelerator for IORT Phys. Med. Biol. 52 6197-6214.

[11] Soriani A, Felici G, Fantini M, Paolucci M, Borla O, Evangelisti G, Benassi M and Strigari L 2010 Radiation protection measurements around a $12 \mathrm{MeV}$ mobile dedicated IORT accelerator Med. Phys. 37 995-1003.

[12] Allison J, Amako K, et al 2006 Geant4 Developments and Applications. IEEE Trans. Nuc.l Sci. 53 270-278.

[13] Geant4.9.5. Available from: http://geant4.web.cern.ch/geant4/

[14] Bjork P, Knoos T and Nilsson P 2004 Measurements of output factors with different detectors types and Monte Carlo calculations of stoppingpower ratios for degraded electron beams Phys. Med. Biol. 49: 44934506.

[15] The COMETA Consortium web site. Available from www.consorziocometa.it

[16] Barbera R, et al 2012 The DECIDE Science Gateway. Journal of Grid Computing (2012), Vol10:689-707. DOI 10.I007/s10723-012-9242-3

[17] The DECIDE Project web site. Available from www.eu-decide.eu

[18] The EUMEDGRID-Support Project web site. Available from www.eumedgrid.eu

[19] The GISELA Project web site. Available from www.gisela-grid.eu

[20] The CHAIN Project web site. Available from www.chain-project.eu

[21] The INDICATE Project web site. Avaialbe from www.indicateproject.eu

[22] The Shibboleth Project web site. Available from shibboleth.internet2.edu

[23] The SimpleSAMLPhp web site. Available from simplesamlphp.org

[24] SAGA Standard specifications. Available from www.gridforum.org/documents/GDF.90.pdf

[25] https://documents.egi.eu/public/ShowDocument?docid=80

[26] https://documents.egi.eu/public/ShowDocument?docid=81 
